\title{
Dunkl generalization of Phillips operators and approximation in weighted spaces
}

\author{
M. Mursaleen ${ }^{1,2,3}$, Md. Nasiruzzaman ${ }^{4}$, A. Kılıçman ${ }^{5^{*}}$ (D) and S.H. Sapar ${ }^{5}$
}

${ }^{\text {*Correspondence: }}$

akilic@upm.edu.my

${ }^{5}$ Department of Mathematics and Institute for Mathematical Research,

Universiti Putra Malaysia, 43400

Serdang, Selangor, Malaysia

Full list of author information is

available at the end of the article

\begin{abstract}
The purpose of this article is to introduce a modification of Phillips operators on the interval $\left[\frac{1}{2}, \infty\right)$ via a Dunkl generalization. We further define the Stancu type generalization of these operators as

$\mathcal{S}_{n, v}^{*}(f ; x)=\frac{n^{2}}{e_{v}\left(n \chi_{n}(x)\right)} \sum_{\ell=0}^{\infty} \frac{\left(n \chi_{n}(x)\right)^{\ell}}{\gamma_{v}(\ell)} \int_{0}^{\infty} \frac{e^{-n t} n_{n}^{\ell+2 v \theta_{\ell}-1} t^{\ell+2 v \theta_{\ell}}}{\gamma_{v}(\ell)} f\left(\frac{n t+\alpha}{n+\beta}\right) \mathrm{d} t, f \in C_{\zeta}\left(R^{+}\right)$, and calculate their moments and central moments. We discuss the convergence results via Korovkin type and weighted Korovkin type theorems. Furthermore, we calculate the rate of convergence by means of the modulus of continuity, Lipschitz type maximal functions, Peetre's $K$-functional and the second order modulus of continuity.
\end{abstract}

MSC: Primary 41A25; 41A36; secondary 33C45

Keywords: Szász operator; Dunkl analogue; Generalization of exponential function; Korovkin type theorem; Modulus of continuity; Order of convergence

\section{Introduction and preliminaries}

The theory of special functions has a long and rich history and a key tool in the study of special functions with reflection symmetries is the Dunkl operator. Various other classes of Dunkl operators have become important, in the first place the trigonometric Dunkl operators of Heckman, Opdam and the Cherednik operators [11]. The aim of this paper is to study a Dunkl type generalization of some approximating operators.

Szász operators [24] provide an extension to Bernstein operators [8] on the interval $[0, \infty)$. In recent years, several authors have studied the Dunkl type generalization of Szász operators (see [13, 14, 16-18, 22, 26]). Recently, several research papers have appeared on Dunkl analogues of different operators (see [2, 3, 5, 9, 19, 25]).

Sucu [23] introduced a Dunkl analogue of Szász operators. That is, for $f \in C[0, \infty), x \geqq 0$, $v \geqq 0$ and $n \in \mathbb{N}$,

$$
\mathcal{S}_{n}^{*}(f ; x):=\frac{1}{e_{v}(n x)} \sum_{\ell=0}^{\infty} \frac{(n x)^{\ell}}{\gamma_{v}(\ell)} f\left(\frac{\ell+2 v \theta_{\ell}}{n}\right),
$$

where $\mathbb{N}$ is the set of all natural numbers and

$$
e_{v}(x)=\sum_{\ell=0}^{\infty} \frac{x^{\ell}}{\gamma_{v}(\ell)}
$$

(c) The Author(s) 2020. This article is licensed under a Creative Commons Attribution 4.0 International License, which permits use sharing, adaptation, distribution and reproduction in any medium or format, as long as you give appropriate credit to the original author(s) and the source, provide a link to the Creative Commons licence, and indicate if changes were made. The images or other third party material in this article are included in the article's Creative Commons licence, unless indicated otherwise in a credit line to the material. If material is not included in the article's Creative Commons licence and your intended use is not permitted by statutory regulation or exceeds the permitted use, you will need to obtain permission directly from the copyright holder. To view a copy of this licence, visit http://creativecommons.org/licenses/by/4.0/. 
The coefficients $\gamma_{v}$ are given as

$$
\gamma_{v}(2 \ell)=\frac{2^{2 \ell} \ell ! \Gamma\left(\ell+v+\frac{1}{2}\right)}{\Gamma\left(v+\frac{1}{2}\right)}, \quad \gamma_{v}(2 \ell+1)=\frac{2^{2 \ell+1} \ell ! \Gamma\left(\ell+v+\frac{3}{2}\right)}{\Gamma\left(v+\frac{1}{2}\right)}
$$

with the recursion

$$
\frac{\gamma_{v}(\ell+1)}{\left(\ell+1+2 v \theta_{\ell+1}\right)}=\gamma_{v}(\ell)
$$

where

$$
\theta_{\ell}= \begin{cases}0 & \text { if } \ell=0,2,4, \ldots \\ 1 & \text { if } \ell=1,3,5, \ldots\end{cases}
$$

Studies on Dunkl type generalizations [20] demonstrate an error estimation to the operators which allow us to have a much faster approximation to the function which is being approximated. Like Bernstein operators which are related to Dunkle type generalization, possibly it can be used for approximate solution of dynamical systems, like [1, 6, 7, 15, 21].

In Sect. 2, we modify the Phillips operators [20] to (2.4) via a Dunkl generalization and further define their Stancu type generalization (2.5). We obtain moments and central moments of these operators. In Sect. 3, we prove some Korovkin type and weighted Korovkin type theorems for operators (2.5). Section 4 is devoted to a study of the rate of convergence by means of the modulus of continuity, Lipschitz type maximal functions, Peetre's $K$-functional and the second order modulus of continuity.

\section{New operators and their moments}

Let $\left\{\chi_{n}(x)\right\}$ be a sequence of nonnegative continuous functions on $[0, \infty)$ as

$$
\chi_{n}(x)=\left(x-\frac{1}{2 n}\right)_{+}, \quad n \in \mathbb{N}
$$

where

$$
\kappa_{+}= \begin{cases}\kappa & \text { if } \kappa \geqq 0, \\ 0 & \text { if } \kappa<0 .\end{cases}
$$

Moreover, suppose

$$
\mathcal{J}_{n, v}(x)=\frac{e_{v}\left(-n \chi_{n}(x)\right)}{e_{v}\left(n \chi_{n}(x)\right)}
$$

For $f \in C_{\zeta}\left(\mathbb{R}^{+}\right)=\left\{f \in C[0, \infty): f(t)=O\left(t^{\zeta}\right), \zeta>n, n \in \mathbb{N}\right\}$, we define

$$
\mathcal{P}_{n, v}(f ; x)=\frac{n^{2}}{e_{v}\left(n \chi_{n}(x)\right)} \sum_{\ell=0}^{\infty} \frac{\left(n \chi_{n}(x)\right)^{\ell}}{\gamma_{v}(\ell)} \int_{0}^{\infty} \frac{e^{-n t} n^{\ell+2 v \theta_{\ell}-1} t^{\ell+2 v \theta_{\ell}}}{\gamma_{v}(\ell)} f(t) \mathrm{d} t, \quad v \geqq 0
$$

where $e_{v}(x), \gamma_{v}$ and $\theta_{\ell}$ are defined as in [23] by (1.2), (1.3) and (1.5), respectively. 
Lemma 2.1 Let $e_{\ell}=t^{\ell-1}, \ell=1,2,3,4,5$ and $\mathcal{J}_{n, v}(x)$ defined by (2.3). Then for $x \geqq 0$, $\mathcal{P}_{n, v}\left(e_{1} ; x\right)=1$ and for any $x \geqq \frac{1}{2 n}$ we have

(1) $\mathcal{P}_{n, v}\left(e_{2} ; x\right)=x+\frac{1}{2 n}$

(2) $\mathcal{P}_{n, v}\left(e_{3} ; x\right)=x^{2}+\frac{1}{n}\left(3+2 v \mathcal{J}_{n, v}(x)\right) x-\frac{1}{4 n^{2}} \mathcal{J}_{n, v}(x)$,

(3) $\mathcal{P}_{n, v}\left(e_{4} ; x\right)=x^{3}+\frac{1}{2 n}\left(15-4 v \mathcal{J}_{n, v}(x)\right) x^{2}$

$$
\begin{aligned}
& +\frac{1}{4 n^{2}}\left(39+16 v+72 v \mathcal{J}_{n, v}(x)\right) x \\
& -\frac{1}{8 n^{3}}\left(7+16 v+68 v \mathcal{J}_{n, v}(x)\right),
\end{aligned}
$$

(4) $\mathcal{P}_{n, v}\left(e_{5} ; x\right)=x^{4}+\frac{1}{n}\left(14+4 v \mathcal{J}_{n, v}(x)\right) x^{3}$

$$
\begin{aligned}
& +\frac{1}{2 n^{2}}\left(99-68 v \mathcal{J}_{n, v}(x)+8 v^{2}\right) x^{2} \\
& +\frac{1}{2 n^{3}}\left(131+294 v \mathcal{J}_{n, v}(x)+96 v^{2}+16 v^{3} \mathcal{J}_{n, v}(x)\right) x \\
& +\frac{1}{16 n^{4}}\left(-367+808 v \mathcal{J}_{n, v}(x)+432 v^{2}+64 v^{3} \mathcal{J}_{n, v}(x)\right) .
\end{aligned}
$$

Remark 2.2 For any $0 \leqq x \leqq \frac{1}{2 n}$, we have $\mathcal{P}_{n, v}\left(e_{2} ; x\right)=\frac{1}{n} ; \mathcal{P}_{n, v}\left(e_{3} ; x\right)=\frac{2}{n^{2}} ; \mathcal{P}_{n, v}\left(e_{4} ; x\right)=\frac{6}{n^{3}}$; $\mathcal{P}_{n, v}\left(e_{5} ; x\right)=\frac{24}{n^{4}}$.

Here we also introduce the Stancu type generalization to the operators defined by (2.4). Thus, for each $f \in C_{\zeta}\left(\mathbb{R}^{+}\right)$the modified version of the operators (2.4) is defined as

$$
\mathcal{S}_{n, v}^{*}(f ; x)=\frac{n^{2}}{e_{v}\left(n \chi_{n}(x)\right)} \sum_{\ell=0}^{\infty} \frac{\left(n \chi_{n}(x)\right)^{\ell}}{\gamma_{v}(\ell)} \int_{0}^{\infty} \frac{e^{-n t} n^{\ell+2 v \theta_{\ell}-1} t^{\ell+2 v \theta_{\ell}}}{\gamma_{v}(\ell)} f\left(\frac{n t+\alpha}{n+\beta}\right) \mathrm{d} t,
$$

where $0 \leq \alpha \leq \beta$. Note that if we take $\alpha=\beta=0$ in (2.5), then the operators $\mathcal{S}_{n, v}^{*}$ reduce to operators defined by (2.4) and if take $\chi_{n}(x)=x$ in $\mathcal{P}_{n, v}$, then we get the operators defined and studied in [20].

Lemma 2.3 For $x \geqq 0, \mathcal{S}_{n, v}^{*}\left(e_{1} ; x\right)=1$ and for $x \geqq \frac{1}{2 n}$, we have

$$
\begin{aligned}
1^{\circ} \quad \mathcal{S}_{n, v}^{*}\left(e_{2} ; x\right)= & \frac{n}{n+\beta} \mathcal{P}_{n, v}\left(e_{2} ; x\right)+\frac{\alpha}{n+\beta}, \\
2^{\circ} \quad \mathcal{S}_{n, v}^{*}\left(e_{3} ; x\right)= & \frac{n^{2}}{(n+\beta)^{2}} \mathcal{P}_{n, v}\left(e_{3} ; x\right)+\frac{2 \alpha n}{(n+\beta)^{2}} \mathcal{P}_{n, v}\left(e_{2} ; x\right)+\frac{\alpha^{2}}{(n+\beta)^{2}}, \\
3^{\circ} \quad \mathcal{S}_{n, v}^{*}\left(e_{4} ; x\right)= & \frac{n^{3}}{(n+\beta)^{3}} \mathcal{P}_{n, v}\left(e_{4} ; x\right)+\frac{3 \alpha n^{2}}{(n+\beta)^{3}} \mathcal{P}_{n, v}\left(e_{3} ; x\right)+\frac{3 \alpha^{2} n}{(n+\beta)^{3}} \mathcal{P}_{n, v}\left(e_{2} ; x\right) \\
& +\frac{\alpha^{3}}{(n+\beta)^{3}},
\end{aligned}
$$




$$
\begin{aligned}
4^{\circ} \quad \mathcal{S}_{n, v}^{*}\left(e_{5} ; x\right)= & \frac{n^{4}}{(n+\beta)^{4}} \mathcal{P}_{n, v}\left(e_{5} ; x\right)+\frac{4 \alpha n^{3}}{(n+\beta)^{4}} \mathcal{P}_{n, v}\left(e_{4} ; x\right)+\frac{6 \alpha^{2} n^{2}}{(n+\beta)^{4}} \mathcal{P}_{n, v}\left(e_{3} ; x\right) \\
& +\frac{4 \alpha^{3} n}{(n+\beta)^{4}} \mathcal{P}_{n, v}\left(e_{2} ; x\right)+\frac{\alpha^{4}}{(n+\beta)^{4}} .
\end{aligned}
$$

Lemma 2.4 For $0 \leqq x \leqq \frac{1}{2 n}$, we have
$(1)^{\circ \circ} \mathcal{S}_{n, v}^{*}\left(e_{2} ; x\right)=\frac{\alpha+1}{n+\beta}$,
$(2)^{\circ \circ} \mathcal{S}_{n, v}^{*}\left(e_{3} ; x\right)=\frac{2+\alpha+\alpha^{2}}{(n+\beta)^{2}}$,
$(3)^{\circ \circ} \mathcal{S}_{n, v}^{*}\left(e_{4} ; x\right)=\frac{6+6 \alpha+3 \alpha^{2}+\alpha^{3}}{(n+\beta)^{3}}$,
$(4)^{\circ \circ} \mathcal{S}_{n, v}^{*}\left(e_{5} ; x\right)=\frac{24+4 \alpha+12 \alpha^{2}+4 \alpha^{3}+\alpha^{4}}{(n+\beta)^{4}}$.

Lemma 2.5 Suppose $\eta_{j}=\left(e_{2}-x\right)^{j}$ for $j=1,2,3,4$, where $e_{2}=t$. Then for $x \geqq \frac{1}{2 n}$ we have

$$
\begin{aligned}
1^{*} \mathcal{S}_{n, v}^{*}\left(\eta_{1} ; x\right)= & \left(\frac{n}{n+\beta}-1\right) x+\frac{1+2 \alpha}{2(n+\beta)}, \\
2^{*} \quad \mathcal{S}_{n, v}^{*}\left(\eta_{2} ; x\right)= & {\left[\frac{n^{2}}{(n+\beta)^{2}}-\frac{2 n}{n+\beta}+1\right] x^{2} } \\
& +\left[\frac{n}{(n+\beta)^{2}}\left(3+2 v \mathcal{J}_{n, v}(x)\right)+\frac{2 \alpha n}{(n+\beta)^{2}}-\frac{2 \alpha+1}{n+\beta}\right] x \\
& +\frac{\alpha+\alpha^{2}}{(n+\beta)^{2}}-\frac{1}{4(n+\beta)^{2}} v \mathcal{J}_{n, v}(x), \\
3^{*} \mathcal{S}_{n, v}^{*}\left(\eta_{4} ; x\right)= & \left.\frac{n^{4}}{(n+\beta)^{4}}-\frac{4 n^{3}}{(n+\beta)^{3}}+\frac{6 n^{2}}{(n+\beta)^{2}}-\frac{4 n}{n+\beta}+1\right] x^{4} \\
& +\left[\frac{n^{3}}{(n+\beta)^{4}}\left(14+4 \alpha+4 v \mathcal{J}_{n, v}(x)\right)\right. \\
& -\frac{2 n^{2}}{(n+\beta)^{3}}\left(15+6 \alpha-4 v \frac{e_{v}(-n \chi(x))}{e_{v}(n \chi(x))}\right) \\
& \left.+\frac{6 n}{(n+\beta)^{2}}\left(3+2 \alpha+2 v \mathcal{J}_{n, v}(x)\right)-\frac{2+4 \alpha}{n+\beta}\right] x^{3} \\
& +\left[\frac{n^{2}}{2(n+\beta)^{4}}\left(99+60 \alpha+8 v^{2}+4(17-4 \alpha) 4 v \mathcal{J}_{n, v}(x)\right)\right. \\
& -\frac{n}{(n+\beta)^{3}}\left(39+16 v+12(3+\alpha)(2+\alpha) v \mathcal{J}_{n, v}(x)\right) \\
& \left.+\frac{1}{2(n+\beta)^{2}}\left(12 \alpha+12 \alpha^{2}+12 \alpha^{2} n^{2}-3 v \mathcal{J}_{n, v}(x)\right)\right] x^{2} \\
& +\left[\frac { n } { 2 ( n + \beta ) ^ { 4 } } \left(131+78 \alpha+16 \alpha^{3}+31 \alpha v+96 v^{2}\right.\right. \\
& \left.+\left(294+144 \alpha+16 v^{2}\right) v \mathcal{J}_{n, v}(x)\right) \\
& \left.+\frac{1}{2(n+\beta)^{3}}\right) \\
&
\end{aligned}
$$




$$
\begin{aligned}
& \left.+\frac{6 \alpha^{2} n}{(n+\beta)^{2}}\left(3+2 v \mathcal{J}_{n, v}(x)\right)\right] x \\
& +\frac{1}{16(n+\beta)^{4}}\left(-367+432 v^{2}+64 v^{3} \mathcal{J}_{n, v}(x)-56 \alpha-128 \alpha v\right. \\
& \left.+16 \alpha^{2}(2 \alpha+1)+(808-544 \alpha) v \mathcal{J}_{n, v}(x)\right)-\frac{3 \alpha^{2}}{2(n+\beta)^{2}} v \mathcal{J}_{n, v}(x)
\end{aligned}
$$

Lemma 2.6 Suppose $\eta_{j}=\left(e_{2}-x\right)^{j}$ for $j=1,2,3,4$, where $e_{2}$ defined in Lemma 2.3. Then for any $0 \leqq x \leqq \frac{1}{2 n}$ we have

$$
\begin{aligned}
(1)^{* *} \quad \mathcal{S}_{n, v}^{*}\left(\eta_{1} ; x\right)= & \frac{\alpha+1}{n+\beta}-x, \\
(2)^{* *} \quad \mathcal{S}_{n, v}^{*}\left(\eta_{2} ; x\right)= & x^{2}-\frac{2(\alpha+1)}{(n+\beta)} x+\frac{2+\alpha+\alpha^{2}}{(n+\beta)^{2}} \\
(3)^{* *} \quad \mathcal{S}_{n, v}^{*}\left(\eta_{4} ; x\right)= & x^{4}-\frac{4(\alpha+1)}{(n+\beta)} x^{3}+\frac{6\left(2+\alpha+\alpha^{2}\right)}{(n+\beta)^{2}} x^{2} \\
& -\frac{4\left(6+6 \alpha+3 \alpha^{2}+\alpha^{3}\right)}{(n+\beta)^{3}} x+\frac{24+24 \alpha+12 \alpha^{2}+4 \alpha^{3}+\alpha^{4}}{(n+\beta)^{4}} .
\end{aligned}
$$

As we can see from Lemmas 2.3 and 2.5, in our analysis of linear operators $\mathcal{S}_{n, v}^{*}(\cdot ; \cdot)$ we have to know the behavior of the function $x \mapsto e_{v}\left(-n \chi_{n}(x)\right) / e_{v}\left(n \chi_{n}(x)\right)$ on $[0, \infty)$. By using (1.2) to (1.5), here we consider the properties of the following ratio:

$$
\mathcal{J}_{n, v}(x)=\frac{e_{v}(-x)}{e_{v}(x)}=e^{-2 x} \frac{\Phi(v, 2 v+1,2 x)}{\Phi(v, 2 v+1,-2 x)}, \quad x \geqq 0,
$$

when $|v|<1 / 2$. Because of $\Phi(0,1, z)=1$, we have $\mathcal{J}_{n, 0}(x)=e^{-2 x}$, and the ratio (2.6) can be expressed in the following form:

$$
\mathcal{J}_{n, v}(x)=\frac{e_{v}(-x)}{e_{v}(x)}=\frac{\Phi(v+1,2 v+1,-2 x)}{\Phi(v, 2 v+1,-2 x)}=\frac{\Phi(v, 2 v+1,2 x)}{\Phi(v+1,2 v+1,2 x)}, \quad x \geqq 0 .
$$

According to (1.2) and (1.3) we get the values of the successive derivatives of the function $e_{v}$ at the origin,

$$
e_{v}^{(k)}(0)=\frac{k !}{\gamma_{v}(k)}, \quad k=0,1,2, \ldots
$$

i.e.,

$$
e_{v}(0)=1, \quad e_{v}^{\prime}(0)=e_{v}^{\prime \prime}(0)=\frac{1}{1+2 v}, \quad e_{v}^{\prime \prime \prime}(0)=e_{v}^{i v}(0)=\frac{3}{(1+2 v)(3+2 v)}, \quad \text { etc. }
$$

\section{Korovkin type approximation}

In the present section the results related to uniform convergence of the operators defined by (2.5) are given via the well-known Korovkin and weighted Korovkin type theorems.

Let $\mathbb{R}^{+}=[0, \infty)$ and $C_{B}\left(\mathbb{R}^{+}\right)$denote the linear normed space with the norm

$$
\|f\|_{C_{B\left(\mathbb{R}^{+}\right)}}=\sup _{x \geqq 0}|f(x)| .
$$


Let

$$
\mathcal{H}:=\left\{f: \lim _{x \rightarrow \infty} \frac{f(x)}{1+x^{2}} \text { exists, } x \in[0, \infty)\right\}
$$

Theorem 3.1 Let the function $f \in C[0, \infty) \cap \mathcal{H}$ and the operators $\mathcal{S}_{n, v}^{*}(\cdot ; \cdot)$ be defined by (2.5). Then

$$
\lim _{n \rightarrow \infty} \mathcal{S}_{n, v}^{*}(f ; x)=f(x)
$$

uniformly on $U$, where $U$ is any compact subset of $[0, \infty)$.

Proof We apply the well-known Korovkin's theorem to prove the uniform convergence of the operators $\mathcal{S}_{n, v}^{*}(\cdot ; \cdot)$. Therefore, for $\ell=1,2,3$, we see $\lim _{n \rightarrow \infty} \mathcal{S}_{n, v}^{*}\left(e_{\ell} ; x\right)=x^{\ell-1}$ uniformly. Therefore, we have

$$
\lim _{n \rightarrow \infty} \mathcal{S}_{n, v}^{*}\left(e_{1} ; x\right)=1 ; \quad \lim _{n \rightarrow \infty} \mathcal{S}_{n, v}^{*}\left(e_{2} ; x\right)=x ; \quad \lim _{n \rightarrow \infty} \mathcal{S}_{n, v}^{*}\left(e_{3} ; x\right)=x^{2}
$$

Hence the result is proved.

We recall the weighted spaces defined by Gadžiev [12]. We write $B_{\sigma}\left(\mathbb{R}^{+}\right)$for the set of all functions such that

$$
|f(x)| \leqq m_{f} \sigma(x)
$$

with $\|f\|_{\sigma}=\sup _{x \geqq 0} \frac{|f(x)|}{\sigma(x)}$, where $m_{f}$ is a constant depending on $f$, and $x \rightarrow \phi(x)$ is a continuous and strictly increasing function such as $\sigma(x)=1+\phi^{2}(x)$ with $\lim _{x \rightarrow \infty} \sigma(x)=\infty$. Let $C_{\sigma}\left(\mathbb{R}^{+}\right)=B_{\sigma}\left(\mathbb{R}^{+}\right) \cap C\left(\mathbb{R}^{+}\right)$. Note that [12] the sequence of positive linear operators $\left\{L_{n}\right\}_{n \geqq 1}$ maps $C_{\sigma}\left(\mathbb{R}^{+}\right)$into $B_{\sigma}\left(\mathbb{R}^{+}\right)$if and only if

$$
\left|L_{n}(\sigma ; x)\right| \leqq K \sigma(x)
$$

with $\sigma(x)=1+\phi^{2}(x), x \in \mathbb{R}^{+}$and $K$ is a positive constant. Let $C_{\sigma}^{0}\left(\mathbb{R}^{+}\right)$be a subset of $C_{\sigma}\left(\mathbb{R}^{+}\right)$ such that

$$
\lim _{x \rightarrow \infty} \frac{f(x)}{\sigma(x)}=k_{f}<\infty
$$

Theorem 3.2 Let $\mathcal{S}_{n, v}^{*}$ be the sequence of positive linear operators acting from $C_{\sigma}\left(\mathbb{R}^{+}\right)$into $B_{\sigma}\left(\mathbb{R}^{+}\right)$such that

$$
\lim _{n \rightarrow \infty}\left\|\mathcal{S}_{n, v}^{*}\left(\varphi^{k}(t) ; x\right)-\varphi^{k}(x)\right\|_{\sigma}=0, \quad k=0,1,2
$$

Then, for all $f \in C_{\sigma}^{0}\left(\mathbb{R}^{+}\right)$, we have

$$
\lim _{n \rightarrow \infty}\left\|\mathcal{S}_{n, v}^{*}(f(t) ; x)-f(x)\right\|_{\sigma}=0
$$


Proof Consider $\varphi(x)=x, \sigma(x)=1+x^{2}$ and

$$
\left\|\mathcal{S}_{n, v}^{*}\left(e_{\ell} ; x\right)-x^{\ell-1}\right\|_{\sigma}=\sup _{x \geqq 0} \frac{\left|\mathcal{S}_{n, v}^{*}\left(e_{\ell} ; x\right)-x^{\ell-1}\right|}{1+x^{2}} .
$$

Then, by Korovkin's theorem, it is easily proved that $\lim _{n \rightarrow \infty}\left\|\mathcal{S}_{n, v}^{*}\left(e_{\ell} ; x\right)-x^{\ell-1}\right\|_{\sigma}=0$, for $\ell=1,2,3$. Hence, for any $f \in C_{\sigma}^{0}\left(\mathbb{R}^{+}\right)$, we get

$$
\lim _{n \rightarrow \infty}\left\|\mathcal{S}_{n, v}^{*}(f(t) ; x)-f(x)\right\|_{\sigma}=0
$$

Theorem 3.3 Let $\mathcal{S}_{n, v}^{*}(\cdot ; \cdot)$ be the operators defined by (2.5). Then for every $f \in C_{\sigma}^{0}\left(\mathbb{R}^{+}\right)$, we have

$$
\lim _{n \rightarrow \infty}\left\|\mathcal{S}_{n, v}^{*}(f ; x)-f\right\|_{\sigma}=0
$$

Proof We prove this theorem in the light of 3.2. Take $f(t)=e_{\ell}$ defined by Lemma 2.3. Then, for any $f(t) \in C_{\sigma}^{0}\left(\mathbb{R}^{+}\right), \mathcal{S}_{n, v}^{*}\left(e_{\ell} ; x\right) \rightarrow x^{\ell-1}(\ell=1,2,3)$ uniformly as $n \rightarrow \infty$. For $\ell=1$, by applying Lemma 2.3 , we get $\mathcal{S}_{n, v}^{*}\left(e_{1} ; x\right)=1$, so that

$$
\lim _{n \rightarrow \infty}\left\|\mathcal{S}_{n, v}^{*}\left(e_{1} ; x\right)-1\right\|_{\sigma}=0 .
$$

Take $\ell=2$ and $x \geqq \frac{1}{2 n}$, we get

$$
\begin{aligned}
\left\|\mathcal{S}_{n, v}^{*}\left(e_{2} ; x\right)-x\right\|_{\sigma} \\
\quad=\sup _{x \geqq 0} \frac{\left|\mathcal{S}_{n, v}^{*}\left(e_{2} ; x\right)-x\right|}{1+x^{2}} \\
\quad=\sup _{x \geqq 0} \frac{\left|\frac{n}{n+\beta} \mathcal{P}_{n, v}\left(e_{2} ; x\right)-x+\frac{\alpha}{n+\beta}\right|}{1+x^{2}} \\
\quad \leq\left(\frac{n}{n+\beta}-1\right) \sup _{x \geqq 0} \frac{x}{1+x^{2}}+\frac{1+2 \alpha}{2(n+\beta)} \sup _{x \geqq 0} \frac{1}{1+x^{2}} .
\end{aligned}
$$

In the case of $0 \leqq x \leqq \frac{1}{2 n}$, we get

$$
\begin{aligned}
& \left\|\mathcal{S}_{n, v}^{*}\left(e_{2} ; x\right)-x\right\|_{\sigma} \\
& \quad=\max _{0 \leqq x \leqq \frac{1}{2 n}} \frac{\left|\mathcal{S}_{n, v}^{*}\left(e_{2} ; x\right)-x\right|}{1+x^{2}} \\
& \quad \leqq \max _{0 \leqq x \leqq \frac{1}{2 n}}\left|\mathcal{S}_{n, v}^{*}\left(e_{2} ; x\right)-x\right| \\
& \leqq \max _{0 \leqq x \leqq \frac{1}{2 n}}\left|\frac{\alpha+1}{n+\beta}-x\right| \\
& \quad=\frac{1}{n+\beta} \max \left\{\alpha+1,\left|\alpha-\frac{\beta}{2 n}\right|\right\} .
\end{aligned}
$$


Then

$$
\lim _{n \rightarrow \infty}\left\|\mathcal{S}_{n, v}^{*}\left(e_{2} ; x\right)-x\right\|_{\sigma}=0
$$

In a similar way if take $\ell=3$ and $x \geqq \frac{1}{2 n}$, we get

$$
\begin{aligned}
\left\|\mathcal{S}_{n, v}^{*}\left(e_{3} ; x\right)-x^{2}\right\|_{\sigma} & \\
= & \sup _{x \geqq 0} \frac{\left|\mathcal{S}_{n, v}^{*}\left(e_{3} ; x\right)-x^{2}\right|}{1+x^{2}} \\
= & \sup _{x \geqq 0} \frac{\left|\frac{n^{2}}{(n+\beta)^{2}} \mathcal{P}_{n, v}\left(e_{3} ; x\right)+\frac{2 \alpha n}{(n+\beta)^{2}} \mathcal{P}_{n, v}\left(e_{2} ; x\right)+\frac{\alpha^{2}}{(n+\beta)^{2}}-x^{2}\right|}{1+x^{2}} \\
\leqq & \left(\frac{n^{2}}{(n+\beta)^{2}}-1\right) \sup _{x \geqq 0} \frac{x^{2}}{1+x^{2}}+\frac{n}{(n+\beta)^{2}}\left(2 \alpha+3+2 v \mathcal{J}_{n, v}(x)\right) \sup _{x \geqq 0} \frac{x}{1+x^{2}} \\
& +\frac{1}{4(n+\beta)^{2}}\left(4 \alpha+4 \alpha^{2}-\mathcal{J}_{n, v}(x)\right) \sup _{x \geqq 0} \frac{1}{1+x^{2}} .
\end{aligned}
$$

In the case of $0 \leqq x \leqq \frac{1}{2 n}$, we get

$$
\begin{aligned}
& \left\|\mathcal{S}_{n, v}^{*}\left(e_{3} ; x\right)-x\right\|_{\sigma} \\
& =\max _{0 \leqq x \leqq \frac{1}{2 n}} \frac{\left|\mathcal{S}_{n, v}^{*}\left(e_{2} ; x\right)-x\right|}{1+x^{2}} \\
& \quad \leqq \max _{0 \leqq x \leqq \frac{1}{2 n}}\left|\mathcal{S}_{n, v}^{*}\left(e_{2} ; x\right)-x\right| \\
& \quad \leqq \max _{0 \leqq x \leqq \frac{1}{2 n}}\left|\frac{2+\alpha+\alpha^{2}}{(n+\beta)^{2}}-x^{2}\right| \\
& =\frac{2+\alpha+\alpha^{2}}{(n+\beta)^{2}}, \\
& \lim _{n \rightarrow \infty}\left\|\mathcal{S}_{n, v}^{*}\left(e_{3} ; x\right)-x^{2}\right\|_{\sigma}=0 .
\end{aligned}
$$

This proves the theorem.

\section{Rate of convergence}

We denote the set of all uniformly continuous functions by $\tilde{C}[0, \infty)$. Let $\tilde{\omega}(f ; \tilde{\delta})$ denote the modulus of continuity of $f \in \tilde{C}[0, \infty)$, i.e.

$$
\tilde{\omega}(f ; \tilde{\delta})=\sup _{\left|x_{1}-x_{2}\right| \leq \delta}\left|f\left(x_{1}\right)-f\left(x_{2}\right)\right| ; \quad x_{1}, x_{2} \in[0, \infty), \tilde{\delta}>0
$$

which satisfies $\lim _{\tilde{\delta} \rightarrow 0+} \tilde{\omega}(f ; \tilde{\delta})=0$, and

$$
\left|f\left(x_{1}\right)-f\left(x_{2}\right)\right| \leq\left(\frac{\left|x_{1}-x_{2}\right|}{\tilde{\delta}}+1\right) \tilde{\omega}(f ; \tilde{\delta}) .
$$


In the light of Lemmas 2.5 and 2.6 we use the notation

$$
\sqrt{\mathcal{S}_{n, v}^{*}\left(\eta_{2} ; x\right)}=\tilde{\delta}_{n, v}(x)
$$

where

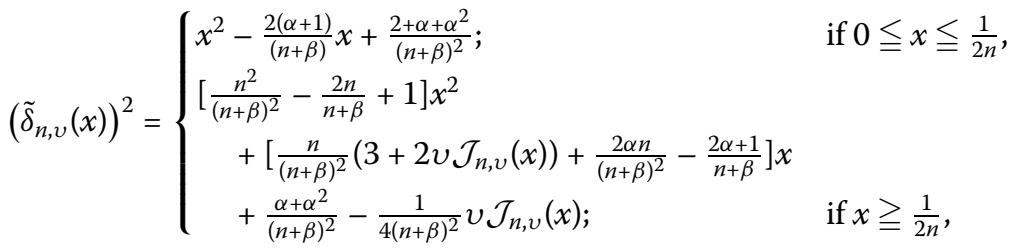

and

$$
\mathcal{J}_{n, v}(x)=\mathcal{J}_{n, v}^{*}\left(n \chi_{n}(x)\right)= \begin{cases}1 ; & \text { if } 0 \leqq x \leqq \frac{1}{2 n} \\ \mathcal{J}_{n, v}^{*}\left(n x-\frac{1}{2}\right) ; & \text { if } x \geqq \frac{1}{2 n}\end{cases}
$$

Theorem 4.1 For any $f \in \tilde{C}[0, \infty)$,

$$
\left|\mathcal{S}_{n, v}^{*}(f ; x)-f(x)\right| \leq 2 \tilde{\omega}\left(f ; \tilde{\delta}_{n, v}(x)\right)
$$

where $\tilde{\delta}_{n, v}(x)$ is defined by (4.4).

Proof Using (4.1) and (4.2), we get

$$
\begin{aligned}
\mathcal{S}_{n, v}^{*}(f ; x)-f(x) & =\mathcal{S}_{n, v}^{*}(f ; x)-f(x) \mathcal{S}_{n, v}^{*}\left(e_{1} ; x\right) \\
& =\mathcal{S}_{n, v}^{*}(f(t)-f(x) ; x) \\
& \leq \mathcal{S}_{n, v}^{*}(|f(t)-f(x)| ; x) .
\end{aligned}
$$

Since $\mathcal{S}_{n, v}^{*}\left(e_{1} ; x\right)=1$, by $(4.2)$ we get

$$
\begin{aligned}
\left|\mathcal{S}_{n, v}^{*}(f ; x)-f(x)\right| & \leq \mathcal{S}_{n, v}^{*}\left(1+\frac{\left|\eta_{1}\right|}{\tilde{\delta}} ; x\right) \tilde{\omega}(f ; \tilde{\delta}) \\
& =\left(1+\frac{1}{\tilde{\delta}} \mathcal{S}_{n, v}^{*}\left(\left|\eta_{1}\right| ; x\right)\right) \tilde{\omega}(f ; \tilde{\delta}) .
\end{aligned}
$$

From the Cauchy-Schwarz inequality we conclude that

$$
\begin{aligned}
\mathcal{S}_{n, v}^{*}\left(\left|\eta_{1}\right| ; x\right) & \leq \mathcal{S}_{n, v}^{*}\left(e_{1} ; x\right)^{\frac{1}{2}} \mathcal{S}_{n, v}^{*}\left(\eta_{2} ; x\right)^{\frac{1}{2}} \\
& =\mathcal{S}_{n, v}^{*}\left(\eta_{2} ; x\right)^{\frac{1}{2}}
\end{aligned}
$$

Therefore,

$$
\left|\mathcal{S}_{n, v}^{*}(f ; x)-f(x)\right| \leq\left(1+\frac{1}{\tilde{\delta}} \mathcal{S}_{n, v}^{*}\left(\eta_{2} ; x\right)^{\frac{1}{2}}\right) \tilde{\omega}(f ; \tilde{\delta}) .
$$

Choose $\tilde{\delta}=\tilde{\delta}_{n, v}(x)=\sqrt{\mathcal{S}_{n, v}^{*}\left(\eta_{2} ; x\right)}$, then we get the result. 
Here we use the usual class of Lipschitz functions and obtain the rate of convergence of the sequence of positive linear operators $\mathcal{S}_{n, v}^{*}(\cdot ; \cdot)(2.5)$. For $\mathcal{L}>0,0<\varrho \leqq 1$ and for the continuous functions $f$ on $[0, \infty)$, the class of Lipschitz functions $\operatorname{Lip}_{\mathcal{L}, \varrho}(f)$ is

$$
\operatorname{Lip}_{\mathcal{L}, \varrho}(f)=\left\{f:\left|f\left(\varsigma_{1}\right)-f\left(\varsigma_{2}\right)\right| \leqq \mathcal{L}\left|\varsigma_{1}-\varsigma_{2}\right|^{\varrho} ; \mathcal{L}>0,0<\varrho \leqq 1\left(\varsigma_{1}, \varsigma_{2} \in[0, \infty)\right)\right\} .
$$

Theorem 4.2 For any $f \in \operatorname{Lip}_{\mathcal{L}, \varrho}$, we have

$$
\left|\mathcal{S}_{n, v}^{*}(f ; x)-f(x)\right| \leqq \mathcal{L}\left(\tilde{\delta}_{n, v}(x)\right)^{\varrho}
$$

where $\tilde{\delta}_{n, v}(x)$ is defined by (4.4).

Proof By the Hölder inequality and (4.6), we get

$$
\begin{aligned}
\left|\mathcal{S}_{n, v}^{*}(f ; x)-f(x)\right| & \leqq\left|\mathcal{S}_{n, v}^{*}(f(t)-f(x) ; x)\right| \\
& \leqq \mathcal{S}_{n, v}^{*}(|f(t)-f(x)| ; x) \\
& \leqq \mathcal{L} \mathcal{S}_{n, v}^{*}\left(\left|\eta_{1}\right|^{\varrho} ; x\right) \\
& \leqq \mathcal{L}\left(\mathcal{S}_{n, v}^{*}\left(e_{1} ; x\right)\right)^{\frac{2-\varrho}{2}}\left(\mathcal{S}_{n, v}^{*}\left(\left|\eta_{1}\right|^{2} ; x\right)\right)^{\frac{e}{2}} \\
& =\mathcal{L}\left(\mathcal{S}_{n, v}^{*}\left(\eta_{2} ; x\right)\right)^{\frac{\rho}{2}} .
\end{aligned}
$$

The space of all continuous and bounded functions on $\mathbb{R}^{+}$is denoted by $C_{B}\left(\mathbb{R}^{+}\right)$and

$$
C_{B}^{2}\left(\mathbb{R}^{+}\right)=\left\{\psi \in C_{B}\left(\mathbb{R}^{+}\right): \psi^{\prime}, \psi^{\prime \prime} \in C_{B}\left(\mathbb{R}^{+}\right)\right\}
$$

The norm on $C_{B}^{2}\left(\mathbb{R}^{+}\right)$is given by

$$
\|\psi\|_{C_{B}^{2}\left(\mathbb{R}^{+}\right)}=\left\|\psi^{\prime \prime}\right\|_{C_{B}\left(\mathbb{R}^{+}\right)}+\left\|\psi^{\prime}\right\|_{C_{B}\left(\mathbb{R}^{+}\right)}+\|\psi\|_{C_{B}\left(\mathbb{R}^{+}\right)},
$$

where the norm for $C_{B}\left(\mathbb{R}^{+}\right)$is

$$
\|\psi\|_{C_{B}\left(\mathbb{R}^{+}\right)}=\sup _{x \geqq 0}|\psi(x)| .
$$

Theorem 4.3 Let $\psi \in C_{B}^{2}\left(\mathbb{R}^{+}\right)$. Then

$$
\left|\mathcal{S}_{n, v}^{*}(\psi ; x)-\psi(x)\right| \leqq \mu_{n, v}(x)\|\psi\|_{C_{B}^{2}\left(\mathbb{R}^{+}\right)},
$$

where $\mu_{n, v}(x)=\tilde{\delta}_{n, v}(x)+\frac{\left(\tilde{\delta}_{n, v}(x)\right)^{2}}{2}$.

Proof By a Taylor series expansion for $\psi \in C_{B}^{2}\left(\mathbb{R}^{+}\right)$we obtain

$$
\begin{aligned}
& \psi(t)=\psi(x)+\psi^{\prime}(x) \eta_{1}+\psi^{\prime \prime}(\varphi) \frac{\eta_{2}}{2} \quad \text { where } \eta_{1}, \eta_{2} \text { are given by (2.5), (2.6), } \\
& |\psi(t)-\psi(x)| \leqq \mathfrak{U}_{1}\left|\eta_{1}\right|+\frac{1}{2} \mathfrak{U}_{2} \eta_{2}
\end{aligned}
$$


where

$$
\begin{aligned}
& \mathfrak{U}_{1}=\sup _{x \geqq 0}\left|\psi^{\prime}(x)\right|=\left\|\psi^{\prime}\right\|_{C_{B}\left(\mathbb{R}^{+}\right)} \leqq\|\psi\|_{C_{B}^{2}\left(\mathbb{R}^{+}\right)}, \\
& \mathfrak{U}_{2}=\sup _{x \geqq 0}\left|\psi^{\prime \prime}(x)\right|=\left\|\psi^{\prime \prime}\right\|_{C_{B}\left(\mathbb{R}^{+}\right)} \leqq\|\psi\|_{C_{B}^{2}\left(\mathbb{R}^{+}\right)} .
\end{aligned}
$$

Therefore,

$$
|\psi(t)-\psi(x)| \leqq\left(\left|\eta_{1}\right|+\frac{1}{2} \eta_{2}\right)\|\psi\|_{C_{B}^{2}\left(\mathbb{R}^{+}\right)} .
$$

By using the linearity of $\mathcal{S}_{n, v}^{*}$ we get

$$
\left|\mathcal{S}_{n, v}^{*}(\psi, x)-\psi(x)\right| \leqq\left(\mathcal{S}_{n, v}^{*}\left(\left|\eta_{1}\right| ; x\right)+\frac{1}{2} \mathcal{S}_{n, v}^{*}\left(\eta_{2} ; x\right)\right)\|\psi\|_{C_{B}^{2}\left(\mathbb{R}^{+}\right)} .
$$

Therefore

$$
\left|\mathcal{S}_{n, v}^{*}(\psi, x)-\psi(x)\right|=\left|\mathcal{S}_{n, v}^{*}(\psi(t)-\psi(x) ; x)\right| \leqq \mathcal{S}_{n, v}^{*}(|\psi(t)-\psi(x)| ; x) .
$$

From the Cauchy-Schwarz inequality

$$
\mathcal{S}_{n, v}^{*}\left(\left|\eta_{1}\right| ; x\right) \leqq\left(\mathcal{S}_{n, v}^{*}\left(\eta_{2} ; x\right)\right)^{\frac{1}{2}}
$$

Thus, we have

$$
\left|\mathcal{S}_{n, v}^{*}(\psi, x)-\psi(x)\right| \leqq\left(\tilde{\delta}_{n, v}(x)+\frac{\left(\tilde{\delta}_{n, v}(x)\right)^{2}}{2}\right)\|\psi\|_{C_{B}^{2}\left(\mathbb{R}^{+}\right)} .
$$

The Peetre's K-functional is a result of potential research work on the approximation process presented by Peetre in 1968. Peetre was able to investigate the interpolation spaces between two Banach spaces and interactions with the real interpolation on the K-functional. For any $f \in C_{B}\left(\mathbb{R}^{+}\right)$, Peetre's well-known $K$-functional property is defined as

$$
\mathcal{K}_{2}(f, \breve{\delta})=\inf \left\{\left(\|f-\psi\|_{C_{B}\left(\mathbb{R}^{+}\right)}+\breve{\delta}\left\|\psi^{\prime \prime}\right\|_{C_{B}^{2}\left(\mathbb{R}^{+}\right)}\right): \psi \in \mathcal{W}^{2}\right\}
$$

where

$$
\mathcal{W}^{2}=\left\{\psi \mid \psi, \psi^{\prime} \text { and } \psi^{\prime \prime} \in C_{B}\left(\mathbb{R}^{+}\right)\right\}
$$

For any $\breve{\delta}>0$ and a positive constant $\mathfrak{C}$ one has $\mathcal{K}_{2}(f ; \breve{\delta}) \leqq \mathfrak{C} \omega_{2}\left(f ; \breve{\delta}^{\frac{1}{2}}\right)$, where

$$
\omega_{2}\left(f ; \breve{\delta}^{\frac{1}{2}}\right)=\sup _{0<h<\check{\delta}^{\frac{1}{2}}} \sup _{t \geqq 0}|f(t+2 h)-2 f(t+h)+f(t)| .
$$

Theorem 4.4 Let $f \in C_{B}\left(\mathbb{R}^{+}\right)$. Then there exists a positive constant $\mathfrak{D}$ such as

$$
\left|\mathcal{S}_{n, v}^{*}(f ; x)-f(x)\right| \leqq 2 \mathfrak{D}\left\{\omega_{2}\left(f ; \sqrt{\frac{\lambda_{n, v}(x)}{2}}\right)+\min \left(1 ; \frac{\lambda_{n, v}(x)}{2}\right)\|f\|_{C_{B}\left(\mathbb{R}^{+}\right)}\right\}
$$

where $\lambda_{n, v}(x)$ is given by 4.3 and $\omega_{2}\left(f ; \frac{\lambda_{n, v}(x)}{2}\right)$ is given by (4.4). 
Proof Take $\psi \in C_{B}^{2}\left(\mathbb{R}^{+}\right)$. Thus we get

$$
\begin{aligned}
\left|\mathcal{S}_{n, v}^{*}(f ; x)-f(x)\right| & \leqq\left|\mathcal{S}_{n, v}^{*}(f-\psi ; x)\right|+\left|\mathcal{S}_{n, v}^{*}(\psi ; x)-\psi(x)\right|+|f(x)-\psi(x)| \\
& \leqq 2\|f-\psi\|_{C_{B}\left(\mathbb{R}^{+}\right)}+\lambda_{n, v}(x)\|\psi\|_{C_{B}^{2}\left(\mathbb{R}^{+}\right)} \\
& =2\left(\|f-\psi\|_{C_{B}\left(\mathbb{R}^{+}\right)}+\frac{\lambda_{n, v}(x)}{2}\|\psi\|_{C_{B}^{2}\left(\mathbb{R}^{+}\right)}\right) .
\end{aligned}
$$

By taking the infimum over all $\psi \in C_{B}^{2}\left(\mathbb{R}^{+}\right)$and by using (4.10), we get

$$
\left|\mathcal{S}_{n, v}^{*}(f ; x)-f(x)\right| \leqq 2 K_{2}\left(f ; \frac{\lambda_{n, v}(x)}{2}\right) .
$$

Now, for an absolute constant $\mathfrak{D}>0$ in [10], we use the following relation:

$$
K_{2}(f ; \breve{\delta}) \leqq \mathfrak{D}\left\{\omega_{2}(f ; \sqrt{\breve{\delta}})+\min (1 ; \breve{\delta})\|f\|_{C_{B}\left(\mathbb{R}^{+}\right)}\right\},
$$

where $\breve{\delta}=\frac{\lambda_{n, v}(x)}{2}$. This completes the proof.

For an arbitrary $f \in C_{\sigma}^{0}\left(\mathbb{R}^{+}\right)$the following weighted modulus of continuity was defined in [4]:

$$
\bar{\Omega}(f ; \hat{\delta})=\sup _{|h| \leq \hat{\delta}, x \geqq 0} \frac{|f(x+h)-f(x)|}{\left(1+x^{2}\right)\left(1+h^{2}\right)},
$$

satisfying

$$
\lim _{\hat{\delta} \rightarrow 0} \bar{\Omega}(f ; \hat{\delta})=0
$$

and

$$
|f(t)-f(x)| \leq 2\left(\frac{|t-x|}{\hat{\delta}}+1\right)\left(1+\hat{\delta}^{2}\right)\left(1+x^{2}\right)\left((t-x)^{2}+1\right) \bar{\Omega}(f ; \hat{\delta}) .
$$

Theorem 4.5 For any $f \in C_{\sigma}^{0}\left(\mathbb{R}^{+}\right)$, we have

$$
\sup _{x \in\left[0, \mathfrak{A}_{n, v}\right)} \frac{\left|\mathcal{S}_{n, v}^{*}(f ; x)-f(x)\right|}{1+x^{2}} \leqq \mathcal{A}\left(1+O\left(\mathfrak{A}_{n, v}\right)\right) \Omega\left(f ; O\left(\sqrt{\mathfrak{A}_{n, v}}\right)\right),
$$

where $\mathcal{A}$ is a positive constant and for $x \geqq \frac{1}{2 n}$

$$
\begin{aligned}
\mathfrak{A}_{n, v}= & \max \left\{\frac{n^{2}}{(n+\beta)^{2}}-\frac{2 n}{n+\beta}+1, \frac{\alpha+\alpha^{2}}{(n+\beta)^{2}}-\frac{1}{4(n+\beta)^{2}} v \mathcal{J}_{n, v}(x),\right. \\
& \left.\frac{n}{(n+\beta)^{2}}\left(3+2 v \mathcal{J}_{n, v}(x)\right)+\frac{2 \alpha n}{(n+\beta)^{2}}-\frac{2 \alpha+1}{n+\beta}\right\}
\end{aligned}
$$

and, for $0 \leqq x \leqq \frac{1}{2 n}$,

$$
\mathfrak{A}_{n, v}=\max \left\{1, \frac{2(\alpha+1)}{(n+\beta)}, \frac{2+\alpha+\alpha^{2}}{(n+\beta)^{2}}\right\} .
$$


Proof We prove it by using (4.13), (4.15) and the Cauchy-Schwarz inequality. We have

$$
\begin{aligned}
& \left|\mathcal{S}_{n, v}^{*}(f ; x)-f(x)\right| \\
& \quad \leqq 2\left(1+\hat{\delta}^{2}\right)\left(1+x^{2}\right) \Omega(f ; \hat{\delta})\left(1+\mathcal{S}_{n, v}^{*}\left(\eta_{2} ; x\right)+\mathcal{S}_{n, v}^{*}\left(\left(1+\eta_{2}\right) \frac{\left|\eta_{1}\right|}{\hat{\delta}} ; x\right)\right) .
\end{aligned}
$$

From Lemmas 2.5, 2.6 we easily conclude that

$$
\begin{aligned}
\mathcal{S}_{n, v}^{*}\left(\eta_{2} ; x\right) & \leqq \mathcal{A}_{1} O\left(\mathfrak{A}_{n, v}\right)\left(1+x+x^{2}\right) \\
& \leqq \mathcal{A}_{2}\left(1+x+x^{2}\right)
\end{aligned}
$$

where $\mathcal{A}_{1}$ and $\mathcal{A}_{2}$ are positive constants, and for $x \geqq \frac{1}{2 n}$

$$
\begin{aligned}
\mathfrak{A}_{n, v}= & \max \left\{\frac{n^{2}}{(n+\beta)^{2}}-\frac{2 n}{n+\beta}+1, \frac{\alpha+\alpha^{2}}{(n+\beta)^{2}}-\frac{1}{4(n+\beta)^{2}} v \mathcal{J}_{n, v}(x),\right. \\
& \left.\frac{n}{(n+\beta)^{2}}\left(3+2 v \mathcal{J}_{n, v}(x)\right)+\frac{2 \alpha n}{(n+\beta)^{2}}-\frac{2 \alpha+1}{n+\beta}\right\}
\end{aligned}
$$

and, for $0 \leqq x \leqq \frac{1}{2 n}$,

$$
\mathfrak{A}_{n, v}=\max \left\{1, \frac{2(\alpha+1)}{(n+\beta)}, \frac{2+\alpha+\alpha^{2}}{(n+\beta)^{2}}\right\} .
$$

By applying the Cauchy-Schwarz inequality we easily see that

$$
\begin{aligned}
\mathcal{S}_{n, v}^{*} & \left(\left(1+\eta_{2}\right) \frac{\left|\eta_{1}\right|}{\delta} ; x\right) \\
& \leqq 2\left(\mathcal{S}_{n, v}^{*}\left(1+\eta_{4} ; x\right)\right)^{\frac{1}{2}}\left(\mathcal{S}_{n, v}^{*}\left(\frac{\eta_{2}}{\hat{\delta}^{2}} ; x\right)\right)^{\frac{1}{2}} .
\end{aligned}
$$

Similarly for the constants $\mathcal{A}_{3}>0$ and $\mathcal{A}_{4}>0$, we have

$$
\begin{aligned}
& \left(\mathcal{S}_{n, v}^{*}\left(1+\eta_{4} ; x\right)\right)^{\frac{1}{2}} \leqq \mathcal{A}_{3}\left(1+x^{2}+x^{3}+x^{4}\right)^{\frac{1}{2}}, \\
& \left(\mathcal{S}_{n, v}^{*}\left(\frac{\eta_{2}}{\hat{\delta}^{2}} ; x\right)\right)^{\frac{1}{2}} \leqq \frac{1}{\hat{\delta}} \mathcal{A}_{4} O\left(\mathfrak{A}_{n, v}\right)^{\frac{1}{2}}\left(1+x+x^{2}\right)^{\frac{1}{2}} .
\end{aligned}
$$

Finally, in view of (4.16), (4.18)-(4.20) and choosing $\hat{\delta}=O\left(\sqrt{\mathfrak{A}_{n, v}}\right)$, and $\mathcal{A}=2\left(1+\mathcal{A}_{2}+\right.$ $2 \mathcal{A}_{3} \mathcal{A}_{4}$ ), we easily are led to the desired result.

\section{Conclusion}

In the present article, we have defined the operators (2.4) via a Dunkl generalization and further defined their Stancu type generalization by (2.5) and obtained their moments and central momemts of these operators. We have proved some Korovkin type and weighted Korovkin type theorems for the operators (2.5). Furthermore, we have studied the rate of convergence by means of the modulus of continuity, Lipschitz type maximal functions, Peetre's $K$-functional and the second order modulus of continuity. It is to be noted that, if 
we take $\alpha=\beta=0$ in (2.5), then the operators $\mathcal{S}_{n, v}^{*}$ reduce to operators defined by (2.4) and if we take $\chi_{n}(x)=x$ in $\mathcal{P}_{n, v}$, then we get the operators defined and studied in [20].

\section{Acknowledgements}

Not applicable.

Funding

No funding available.

\section{Availability of data and materials}

Data sharing is not applicable to this article as no datasets were generated or analyzed during the current study.

\section{Competing interests}

The authors declare that they have no competing interests.

\section{Authors' contributions}

The authors contributed equally and significantly in writing this paper. All authors read and approved the final manuscript.

\section{Author details}

${ }^{1}$ Department of Medical Research, China Medical University Hospital, China Medical University (Taiwan), Taichung, Taiwan. ${ }^{2}$ Department of Mathematics, Aligarh Muslim University, Aligarh 202002, India. ${ }^{3}$ Department of Computer Science and Information Engineering, Asia University, Taichung, Taiwan. ${ }^{4}$ Department of Mathematics, Faculty of Science, University of Tabuk, P.O. Box 741, Tabuk 71491, Saudi Arabia. ${ }^{5}$ Department of Mathematics and Institute for Mathematical Research, Universiti Putra Malaysia, 43400 Serdang, Selangor, Malaysia.

\section{Publisher's Note}

Springer Nature remains neutral with regard to jurisdictional claims in published maps and institutional affiliations.

Received: 16 March 2020 Accepted: 6 July 2020 Published online: 17 July 2020

\section{References}

1. Alijani, Z., Baleanu, D., Shiri, B., Wu, G.-C.: Spline collocation methods for systems of fuzzy fractional differential equations. Chaos Solitons Fractals 131, 109510 (2020)

2. Alotaibi, A.: Approximation by a generalized class of Dunkl type Szász operators based on post quantum calculus J. Inequal. Appl. 2019, 241 (2019)

3. Alotaibi, A., Nasiruzzaman, M., Mursaleen, M.: A Dunkl type generalization of Szasz operators via post-quantum calculus. J. Inequal. Appl. 2018, 287 (2018)

4. Atakut, C., Ispir, N.: Approximation by modified Szász-Mirakjan operators on weighted spaces. Proc. Indian Acad. Sci. Math. Sci. 112, 571-578 (2002)

5. Atakut, C.., Karateke, S., Büyükyazıcı, I.: On approximation process by certain modified Dunkl generalization of Szász-beta type operators. J. Math. Comput. Sci. 19, 9-18 (2019)

6. Baleanu, D., Shiri, B.: Collocation methods for fractional differential equations involving non-singular kernel. Chaos Solitons Fractals 116, 136-145 (2018)

7. Baleanu, D., Shiri, B., Srivastava, H.M., Al Qurashi, M.: A Chebyshev spectral method based on operational matrix for fractional differential equations involving non-singular Mittag-Leffler kernel. Adv. Differ. Equ. 2018, Article ID 353 (2018)

8. Bernstein, S.N.: Démonstration du théoréme de Weierstrass fondée sur le calcul des probabilités. Commun. Soc. Math. Kharkow 2(13), 1-2 (1912)

9. Çekim, B., Kantar, Ū.D., Yüksel, I.: Dunkl generalization of Szász beta-type operators. Math. Methods Appl. Sci. 40 7697-7704 (2017)

10. Ciupa, A.: A class of integral Favard-Szász type operators. Stud. Univ. Babeş-Bolyai, Math. 40(1), 39-47 (1995)

11. Dunkl, C.F., Xu, Y.: Orthogonal Polynomials of Several Variables. Cambridge Univ. Press, Cambridge (2001)

12. Gadžiev, A.: A problem on the convergence of a sequence of positive linear operators on unbounded sets, and theorems that are analogous to P.P. Korovkin's theorem. Dokl. Akad. Nauk SSSR 218, 1001-1004 (1974) (Russian)

13. İçōz, G., Çekim, B.: Dunkl generalization of Szász operators via q-calculus. J. Inequal. Appl. 2015, 284 (2015)

14. İçōz, G., Çekim, B.: Stancu type generalization of Dunkl analogue of Szász-Kamtrovich operators. Math. Methods Appl. Sci. 39(7), 1803-1810 (2016)

15. Ma, C.Y., Shiri, B., Wu, G.-C., Baleanu, D.: New signal smoothing equations with short memory and variable order. Optik $218,164507(2020)$

16. Milovanovic, G.V., Mursaleen, M., Nasiruzzaman, Md.: Modified Stancu type Dunkl generalization of Szasz-Kantorovich operators. Rev. R. Acad. Cienc. Exactas Fís. Nat., Ser. A Mat. 112, 135-151 (2018)

17. Mursaleen, M., Nasiruzzaman, Md., Alotaibi, A.: On modified Dunkl generalization of Szász operators via q-calculus. J. Inequal. Appl. 2017, 38 (2017)

18. Nasiruzzaman, Md., Mukheimer, A., Mursaleen, M.: Approximation results on Dunkl generalization of Phillips operators via q-calculus. Adv. Differ. Equ. 2019, 244 (2019)

19. Nasiruzzaman, Md., Mukheimer, A., Mursaleen, M.: A Dunkl type generalization of Szász-Kantorovich operators via post-quantum calculus. Symmetry 11, 232 (2019) 
20. Nasiruzzaman, Md., Nadeem, R.: A generalized Dunkl type modification of Phillips operators. J. Inequal. Appl. 2018, $323(2018)$

21. Shiri, B., Baleanu, D.: Numerical solution of some fractional dynamical systems in medicine involving non-singular kernel with vector order. Results Nonlinear Anal. 2(4), 160-168 (2019)

22. Srivastava, H.M., Mursaleen, M., Alotaibi, A., Nasiruzzaman, Md., Al-Abied, A.: Some approximation results involving the q-Szasz-Mirakjan-Kantorovich type operators via Dunkl's generalization. Math. Methods Appl. Sci. 40(15), 5407-5682 (2017)

23. Sucu, S.: Dunkl analogue of Szász operators. Appl. Math. Comput. 244,42-48 (2014)

24. Szász, O.: Generalization of S. Bernstein's polynomials to the infinite interval. J. Res. Natl. Bur. Stand. 45, $239-245$ (1950)

25. Taşdelen, F., Söylemez, D., Aktaş, R.: Dunkl-gamma type operators including Appell polynomials. Complex Anal. Oper. Theory 13, 3359-3371 (2019)

26. Wafi, A., Rao, N.: Szász-Durrmeyer operators based on Dunkl analogue. Complex Anal. Oper. Theory 12(7), 1519-1536 (2018)

Submit your manuscript to a SpringerOpen ${ }^{\circ}$ journal and benefit from:

- Convenient online submission

- Rigorous peer review

- Open access: articles freely available online

- High visibility within the field

- Retaining the copyright to your article

Submit your next manuscript at $\boldsymbol{~ s p r i n g e r o p e n . c o m ~}$ 